Publicación cuatrimestral. Vol. 5, No. 5 (Septiembre-Diciembre), Año 2021. Pág. 1-14

LA VINCULACIÓN CON LA SOCIEDAD DE LA UNESUM

\title{
LA VINCULACIÓN CON LA SOCIEDAD DE LA UNESUM Y SU APORTE EN LOS ESCENARIOS CAFETALEROS DE JIPIJAPA, PERIODO 2016 - 2018
}

\author{
AUTORES: Tomas Robert Fuentes Figueroa ${ }^{1}$ \\ Kevin José Delgado Pinargote ${ }^{2}$ \\ William Ausberto Merchán García ${ }^{3}$ \\ Diana Valverde Jalca ${ }^{4}$ \\ Yhony Alfredo Valverde Lucio ${ }^{5}$
}

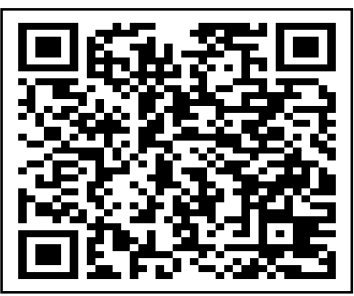

DIRECCIÓN PARA CORRESPONDENCIA: (tomas.fuentes@unesum.edu.ec)

Fecha de recepción: 02/01/2021

Fecha de aceptación: 28/08/2021

\section{RESUMEN}

La vinculación con la sociedad en la Universidad Estatal del Sur de Manabí, a través de la ejecución de proyectos de servicios comunitarios, propicia generar impacto social y productivo sostenible, a fin de impulsar el desarrollo en los escenarios sociales permanentes identificados en el territorio. Los escenarios cafetaleros, son la integración de varias familias caficultoras ubicadas en el área rural del cantón Jipijapa, cuyas pequeñas fincas (UPAs) se caracterizan por su diversificación productiva, teniendo al cultivo de café como la actividad económica más importante. La investigación tuvo como objetivo general, el determinar el impacto de la vinculación con la sociedad en los escenarios cafetaleros de la UNESUM durante el periodo 2016-2018. Se aplicó un enfoque metodológico cuantitativo y cualitativo de carácter descriptivo e inferencial, la información de sustento fue de fuentes primarias y secundarias, que oportunamente fue emitida por los caficultores al responder encuestas en los tiempos señalados. Los datos no presentaron distribución normal, lo que dio lugar a la aplicación de estadística no paramétrica, aplicando la prueba de U Mann Whitney cuyo p valor $<0,01$, definió diferencias altamente significativas, deduciendo que la intervención de la UNESUM por medio del proyecto de investigación, si insidio en el incremento productivo de los caficultores involucrados; el incremento productivo en el periodo de estudio, a nivel agrícola representó un incremento del 292,2 \%; se considera diversos productos agrícolas, entre los que destacan el plátano, maíz,

\footnotetext{
1 Magister en Gerencia Educativa; Diplomado en Autoevaluación y Acreditación Universitaria; Ingeniero Agropecuario; Profesor de Segunda Enseñanza; Docente de la Facultad Ciencias Naturales y de la Agricultura, Universidad Estatal del sur de Manabí, Jipijapa, Ecuador. tomas.fuentes@unesum.edu.ec

2 Ingeniero Agropecuaria, Universidad Estatal del Sur de Manabí, Jipijapa, Ecuador, delgadokevin0555@unesum.edu.ec

${ }^{3}$ Magister en Finanzas y Comercio Internacional; Ingeniero Industrial, Docente de la Facultad Ciencias Naturales, Universidad Estatal del sur de Manabí, Jipijapa, Ecuador. William.merchan@unesum.edu.ec

${ }^{4}$ Egresada de la Carrera de Ingeniería Forestal de la Universidad Estatal del sur de Manabí, Jipijapa, Ecuador. diana.valverde@unesum.edu.ec.

${ }^{5}$ Magister en Gestión de Proyectos Socio Productivos, Ingeniero Agropecuario, Docente Titular, Facultad Ciencias Naturales, Universidad Estatal del sur de Manabí, Jipijapa, Ecuador. yhony.valverde@unesum.edu.ec
} 
naranja, además del café, este último rubro tiene un incremento del de 288,46 \%.

Palabras clave: comunitario; escenarios; impacto; vinculación.

\title{
THE LINK WITH THE UNESUM SOCIETY AND ITS CONTRIBUTION IN THE COFFEE-GROWING SCENARIOS OF JIPIJAPA, PERIOD 2016 - 2018
}

\begin{abstract}
The link with society at the Universidad Estatal del Sur de Manabí, through the implementation of community service projects, is conducive to generate sustainable social and productive impact, in order to promote development in the permanent social scenarios identified in the territory. The coffee-growing scenarios are the integration of several coffee-growing families located in the rural area of the Jipijapa canton, whose small farms (UPAs) are characterized by their productive diversification, with coffee cultivation as the most important economic activity. The general objective of the research was to determine the impact of the linkage with society in the coffeegrowing scenarios of UNESUM during the period 2016-2018. A quantitative and qualitative methodological approach of descriptive and inferential character was applied, the supporting information was from primary and secondary sources, which was timely issued by the coffee growers by answering surveys at the indicated times. The data did not present normal distribution, which gave rise to the application of non-parametric statistics, applying the Mann Whitney $U$ test whose $\mathrm{p}$ value $<0.01$, defined highly significant differences, deducing that the intervention of UNESUM through the research project did have an impact on the productive increase of the coffee growers involved; The increase in production during the study period, at the agricultural level, represented an increase of 292.2\%; various agricultural products are considered, among which bananas, corn, oranges, and coffee stand out, this last item has an increase of $288.46 \%$.
\end{abstract}

Key words: bonding; community; impact; scenarios.

\section{INTRODUCCIÓN}

El café cultivo se encuentra entre los principales productos agrícolas del país, se ubica entre los 10 de mayor superficie cultivada (Andrade, 2017), Pozo (2014) indica, que la producción de café es importante para la economía ecuatoriana, ya que aporta divisas al Estado, aportando ingresos para las familias que cultivan café, beneficia a los actores de la cadena productiva del café.

La vinculación con la sociedad en la Universidad Estatal del Sur de Manabí "UNESUM", constituye una de las funciones sustantivas del que hacer universitario articulado a la docencia e investigación. Esta relacionado entre lo que hace la universidad y lo que la sociedad espera de ésta, de tal manera que, a través de la ejecución de proyectos de servicios comunitarios, se genere impacto social y productivo en el tiempo e impulse el desarrollo sostenible en los escenarios sociales permanentes identificados en el territorio.

Un escenario social permanente, es un grupo de personas, una comunidad, una asociación, una familia, una institución pública e incluso una institución privada que se relacionan entre sí en un área geográficamente específica y cuyos miembros comparten actividades de interés económico, cultural y social e intereses comunes (Modelo de Vinculación-UNESUM 2017).

Los escenarios cafetaleros, son la integración de varias familias caficultoras ubicadas en el área rural del cantón Jipijapa que poseen pequeñas fincas (UPAs) diversificadas, teniendo como referencia la producción de café como la actividad económica más importante, se complementan

2 UNESUM-Ciencias. Publicación cuatrimestral. Vol. 5, Año 2021, No. 5 (Septiembre-Diciembre) 
Publicación cuatrimestral. Vol. 5, No. 5 (Septiembre-Diciembre), Año 2021. Pág. 1-14

LA VINCULACIÓN CON LA SOCIEDAD DE LA UNESUM

con otras producciones agrícolas para el autoconsumo como plátano, maíz, naranja, y en la producción pecuaria las aves y cerdos.

La investigación tuvo como objetivo, el determinar el impacto de la vinculación con la sociedad en los escenarios cafetaleros de la UNESUM durante el perdió 2016-2018, y para esto describió el comportamiento productivo de manera integral de los escenarios cafetaleros.

\section{DESARROLLO}

Las universidades y escuelas politécnicas, que son instituciones académicas que brindan formación en áreas profesionales y disciplinas científicas y tecnológicas; desarrollan investigación social, científica y tecnológica de manera permanente y mantienen programas de vinculación con la colectividad, orientados al desarrollo social, económico, político y cultural del país. Consejo de Aseguramiento de la Calidad de la Educación Superior CACES (2020)

La vinculación con la sociedad hace referencia a la planificación, ejecución y difusión de actividades que garantizan la participación efectiva en la sociedad y responsabilidad social de las instituciones del Sistema de Educación Superior con el finde contribuir a la solución de las necesidades y problemáticas del entorno desde el ámbito académico e investigativo. La vinculación con la sociedad deberá articularse al resto de funciones sustantivas, oferta académica, dominios académicos, investigación, formación y extensión de las IES en cumplimiento del principio de pertinencia. (Reglamento al régimen académico, 2019).

La Red Ecuatoriana Universitaria de Vinculación con la Colectividad "REUVIC", se constituye de conformidad con las leyes ecuatorianas como una entidad de derecho privado, sin fines de lucro y con personería jurídica y se rige por su estatuto y por los reglamentos internos que la determinan. La REUVIC está integrada por las Universidades, Escuelas Politécnicas e Institutos Técnicos y Tecnológicos Superiores del Ecuador y/o extranjeros. Su principal atención se centra en el trabajo conjunto y colaborativo en alianzas estratégicas con instituciones de carácter público o privado que forman parte del Sistema de Educación Superior, así como la sociedad civil organizada, para la promoción de programas y proyectos de Vinculación con la Sociedad. (Borroto, 2018)

Son objetivos estratégicos de la REUVIC.

a) Promover el desarrollo de la Vinculación con la Sociedad, como función sustantiva que articula el quehacer universitario, mediante programas y proyectos regionales y locales.

b) Promover el trabajo conjunto y colaborativo de las IES, aprovechando las capacidades y fortalezas de los centros de educación superior y los actores sociales comprometidos.

c) Impulsar la formulación e implementación de proyectos articulados y o conjuntos que conlleven al desarrollo local, regional y nacional; fortaleciendo la movilización y generación de capacidades dentro y fuera del país.

d) Contribuir a la definición de: políticas, lineamientos y estrategias para el desarrollo de la vinculación con la sociedad.

e) Promover escenarios de capacitación conjunta en la perspectiva de mejorar los procesos de la vinculación con la sociedad. (Borroto, 2018)

La Universidad Estatal del Sur de Manabí cuenta con un Modelo de vinculación por escenarios sociales; considerando el escenario es un "grupo de personas que se relacionan entre sí en un área geográficamente específica y cuyos miembros comparten actividades e intereses comunes, donde pueden o no cooperar para la solución de los problemas comunes. Es una comunidad económico- 
social integrada que responde al concepto de desarrollo que realiza actividades de interés económico, cultural y social. Esto permite: Concretar los objetivos, misión y visión en los escenarios, Tener claro acciones y resultados. Mayor compromiso. Más identidad. Más sentido de pertenencia. (Modelo de vinculación UNESUM, 2018)

Un escenario social es para la UNESUM, el espacio permanente de trabajo en el que se realizan diversas acciones tendientes a mejorar su calidad de vida, a partir de oportunidades de índole socio - académicas. Un escenario puede ser: una organización, una comunidad, un gobierno autónomo parroquial, cantonal, una unidad educativa, una familia, una asociación campesina, emprendimientos familiares, etc. (Modelo de vinculación UNESUM, 2018)

Para los ecuatorianos, la caficultura tiene importancia en los órdenes: económico, social, ambiental, institucional y salud humana. En lo económico, constituye una fuente de divisas e ingresos para los actores de las cadenas del café. En lo social, en las cadenas del café se involucran muchas etnias y pueblos en 23 de las 24 provincias de Ecuador, dispersos en un amplio tejido social. En lo ambiental, se cultiva básicamente en sistemas agroforestales, en una amplia diversidad de suelos y climas, contribuyendo a la conservación de la fauna y flora nativas. (Ponce et al., 2018)

Santistevan et al., (2014), en su estudio sobre la caracterización de las fincas cafetaleras, concluye que, en la localidad de Jipijapa, las fincas son muy diversificadas, la producción de café es la actividad más importante, pero no es la única actividad; sino que se complementa con producción agropecuaria para el autoconsumo. Los bajos rendimientos muestran que hay necesidad de una mejora importante en el manejo técnico del cultivo de café. Además, el déficit de servicios básicos y de infraestructura, también es significativo y su solución debería abordarse a la mayor brevedad posible. La mejora en cada uno estos aspectos conllevaran a la mejor y mayor competitividad del sector cafetalero en la localidad de Jipijapa.

\section{Materiales y métodos}

La investigación aplicó un enfoque cuantitativo y cualitativo de carácter descriptivo e inferencial, la información de sustentación fue de fuentes primarias y secundarias, que posteriormente se analizaron estadísticamente, con el fin de definir la relación entre variables de estudio y respuesta respectivamente, generando gráficos y análisis de los resultados obtenidos.

De igual manera la investigación plantea una prueba de hipótesis de correlación de variables, lo que induce el uso de estadística no paramétrica aplicando la prueba de U Mann Whitney, también conocido como Wilcoxon, test no paramétrico que contrasta si dos muestras proceden de poblaciones equidistribuidas.

De lo indicado, el test de Mann-Whitney-Wilcoxon contrasta que la probabilidad de que una observación de la población X supere a una observación de la población Y es igual a la probabilidad de que una observación de la población Y supere a una de la población X. Es decir, que los valores de una población no tienden a ser mayores que los de otra.

\section{RESULTADOS}

Se aplicó una encuesta a 60 productores cafetaleros, llamados escenarios, denominados así en el modelo de vinculación de la UNESUM, otorga este nombre a una familia, una comunidad o varias comunidades, una parroquia, etc. que son parte de la labor vinculatoria de la universidad. Interpretación de tabla 1 . Se indagó sobre la edad de los productores cafetaleros, y como se puede observar, la mayoría de ellos (53 \%) tiene más de 60 años, siendo apenas un 13,5\% de

4 UNESUM-Ciencias. Publicación cuatrimestral. Vol. 5, Año 2021, No. 5 (Septiembre-Diciembre) 
caficultores menores de 40 años, situación que es preocupante para este sector, considerando que la experiencia permite inferir posibles abandonos de fincas.

Tabla 1. Edad de jefes de hogar de escenarios cafetaleros

\begin{tabular}{lccc}
\hline & Edad & Frecuencia & \% \\
\hline 20 a 30 & & 3 & 5,00 \\
31 a 40 & 8 & 13,00 \\
41 a 50 & & 13 & 22,00 \\
51 a 60 & & 4 & 7,00 \\
más de 61 & Total & 60 & 53,00 \\
& & & $100 \%$ \\
\hline
\end{tabular}

Elaborado por: Autores del artículo

Interpretación de la figura 1. En lo que respecta al sexo de los jefes de hogar, se identifica que en los escenarios cafetaleros lideran los procesos productivos agrícolas los hombres con el 63,33\%, particularmente en la producción de café; el sexo femenino con el 36,67\%. Este resultado determina que en nuestro sector rural aún existe predominancia del patriarcado.

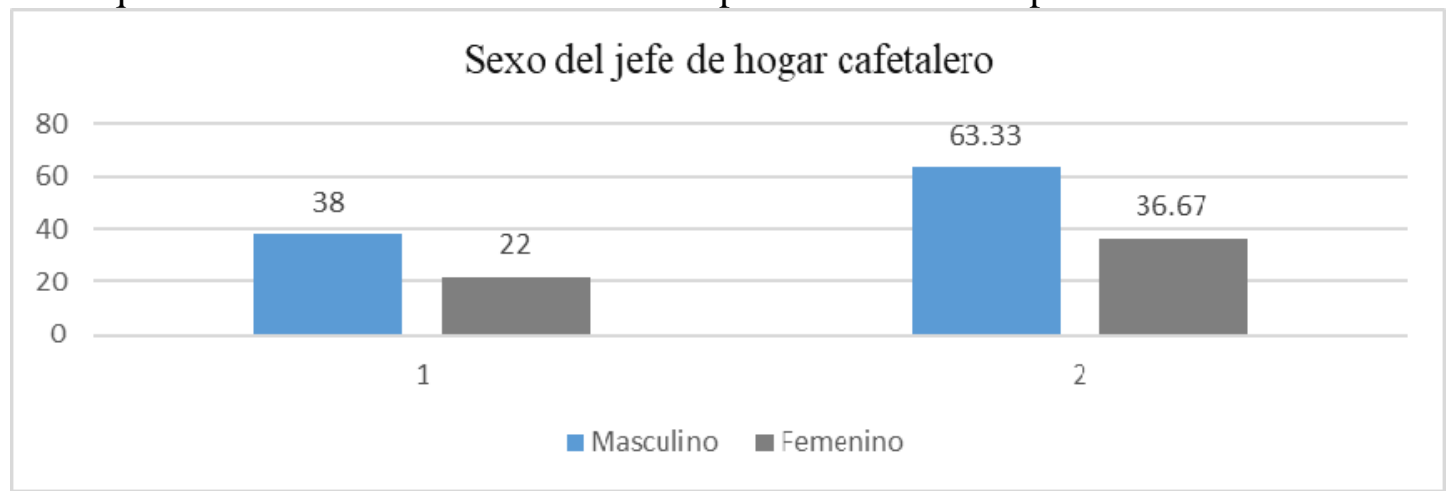

Figura 1. Sexo de jefes de hogar cafetaleros

Elaborado por: Autores del artículo

Interpretación de la figura2. Entre los aspectos sociales a tomar en cuenta, estuvo la escolaridad, identificándose que el $46 \%$ solo ha aprobado la primaria; un 8\% de productores no pudo concluir la primaria; el 19 \% concluyo la secundaria; el 8 \% no concluyó la secundaria; un 2 \% ingreso a la universidad y no lo concluyó; el $11 \%$ de los encuestados indican tener título de tercer nivel; también se identifica un $2 \%$ de analfabetismo.

Las particularidades sobre el nivel educativo, dan pauta para tomar acciones orientadas a capacitar a los caficultores, usando herramientas andragógicas, que permitan hacer amigable el aprendizaje, haciendo de esta manera valido el proceso vinculatorio de la universidad con la sociedad. 


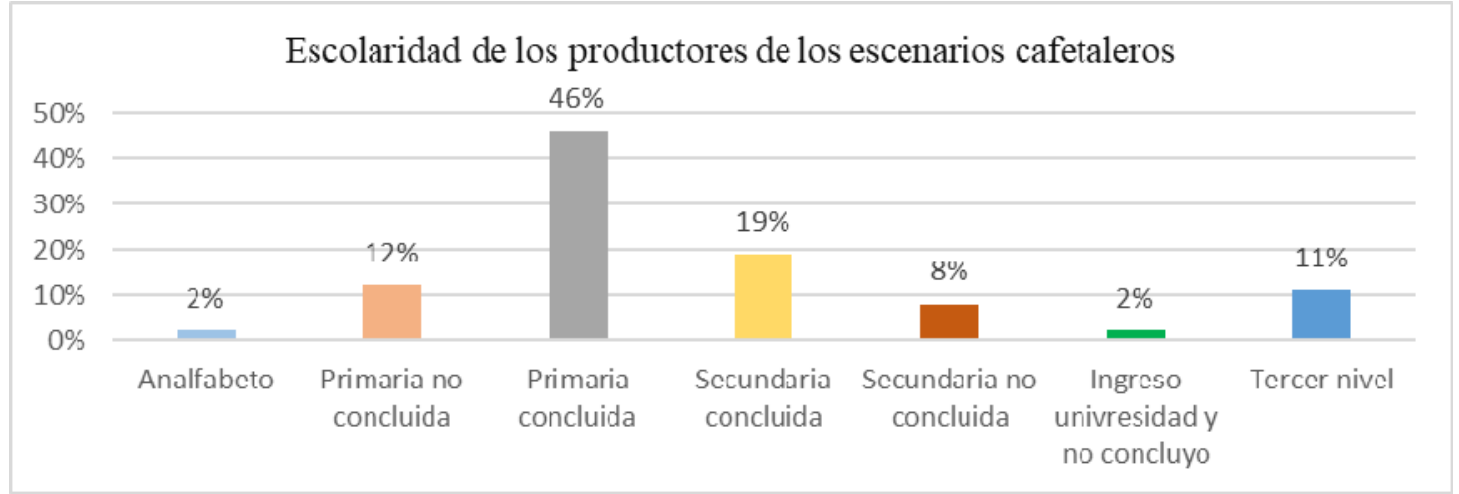

Figura 2. Escolaridad de escenarios cafetaleros

Elaborado por: Autores del artículo

Interpretación de la figura 3. Un aspecto importante de analizar es la unión de la familia en el sector rural y particularmente las del sector cafetalero del sur manabita, donde es apreciable la inexistencia de divorcios, lo que pudiese ocurrir por aspectos culturales y hasta religioso, donde se considera a la familia como una unión sagrada. Se identifica que el 57\% están casados y un $23 \%$ son solteros.

Estado civil de los jefes de hogar de los escenarios cafetaleros

- Soltero $\quad$ Unión Libre $\quad$ Casado Viudo $\quad$ Divorciado

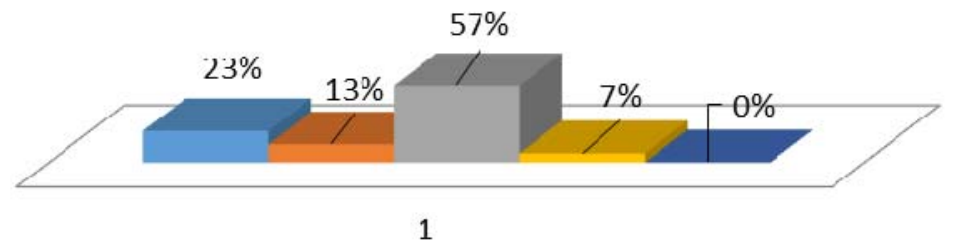

Figura 3. Estado civil de los jefes de hogar de los escenarios cafetaleros Elaborado por: Autores del artículo

Interpretación de tabla 2. Se analizó la calidad de agua que ocupan los escenarios cafetaleros para el consumo humano. El 42 \% cuenta con agua entubada captada desde vertientes naturales; el 32 $\%$ consumen agua de vertientes;el $18 \%$ dispone de agua de pozo; el 7\% dispone de agua entubada procedente de pozos comunitarios; mientras que solo el $10 \%$ indicó disponer de agua potable. Los escenarios no tienen disponibilidad de agua para riego por ser escasa y además no existe infraestructura de riego.

El recurso agua en la zona sur manabita es un limitante en lo que a producción agropecuaria se refiere, este recurso solo está disponible de forma condicionada para el consumo humano.

6 UNESUM-Ciencias. Publicación cuatrimestral. Vol. 5, Año 2021, No. 5 (Septiembre-Diciembre) 
Tabla 2. Calidad del agua

\begin{tabular}{lcc}
\hline \multicolumn{1}{c}{ Clase } & Frecuencia & \% \\
\hline Agua potable & 6 & $10 \%$ \\
Agua de vertiente no entubada & 14 & $23 \%$ \\
Agua pozo no entubada & 11 & $18 \%$ \\
Agua de rio no entubada & 0 & $0 \%$ \\
Agua de vertiente entubada & 25 & $42 \%$ \\
Agua de pozo entubado & 4 & $7 \%$ \\
\multicolumn{1}{c}{ Total } & 60 & $100 \%$ \\
\hline
\end{tabular}

Elaborado por: Autores del artículo

Interpretación de tabla 3. En lo que respecta a la vivienda, el 35 \% tiene casas de cemento; otro $35 \%$ tiene casas de madera y caña, y el 30\% dispone de casas mixtas construido de cemento y madera.

Tabla 3. Tipo de vivienda

\begin{tabular}{lcc}
\hline \multicolumn{1}{c}{ Clase } & Frecuencia & \% \\
\hline Cemento & 21 & $35 \%$ \\
Madera/caña & 21 & $35 \%$ \\
Cemento y madera & 18 & $30 \%$ \\
\multicolumn{1}{c}{ Total } & 60 & $100 \%$ \\
\hline
\end{tabular}

Elaborado por: Autores del artículo

Interpretación de tabla4. Es oportuno, no solo saber del tipo de viviendas que dispone el caficultor, también se identificó el estado de ellas, y como se puede observar el $47 \%$ de los caficultores tiene viviendas en estado regular;23 \% en malas condiciones; y solo un $30 \%$ tiene su vivienda en buenas condiciones. Esto implica brindar atención al sector en este aspecto, más aún cuando la vivienda es parte del análisis de indicadores de pobreza.

Tabla 4. Estado de la vivienda

\begin{tabular}{|c|c|c|}
\hline Clase & Frecuencia & $\%$ \\
\hline Buenas condiciones & 18 & $30 \%$ \\
\hline Regular & 28 & $47 \%$ \\
\hline Malas condiciones & 14 & $23 \%$ \\
\hline Total & 60 & $100 \%$ \\
\hline
\end{tabular}

Elaborado por: Autores del artículo

Sobre la pregunta, si cuentan o no con servicio de recolección de la basura, el 82 \% indico no disponer de este servicio, entendiendo que la mayoría de los caficultores, la quema, entierra y/o arroja a las quebradas, generando de esta manera problemas ambientales.

Entre otros aspectos analizados en el estudio, se encuentran: la disponibilidad de servicio eléctrico y telefónico, en ambos aspectos la totalidad de productores indican que sí disponen de estos servicios. 
En cuanto al servicio de alcantarillado, como es de conocimiento general, no se dispone en los sectores rurales de este importante servicio; por lo que el productor construye sus letrinas, acompañadas de pozos sépticos.

Interpretación de tabla5. Es oportuno mencionar que, aunque el análisis obedece a aspectos productivos relacionados al café, se efectuó una revisión general de otros rubros que significan potenciales ingresos para las familias caficultoras, más aún cuando a estos rubros también son parte considerados en las actividades de vinculación

Como se puede observar, el incremento productivo en el periodo de estudio, a nivel agrícola es significativo, y está representado por un incremento del 292,2 \%; y considera diversos productos agrícolas, entre los que destacan el plátano, maíz, naranja, además del café, este último rubro tiene un incremento del de $288,46 \%$. Los resultados indican un cambio a nivel de ingresos, que resulta motivante para el productor, esto deduce que la intervención de la Universidad, por medio de proyectos de vinculación ha motivado al productor a mejorar el manejo del cultivo de café.

Tabla 5. Escolaridad de escenarios cafetaleros

\begin{tabular}{lccc}
\hline \multicolumn{1}{c}{ Rubros } & Año 2016 & Año 2018 & Incremento \% \\
\hline Café & $\$ 5.668,00$ & $\$ 16.350,00$ & 288,46 \\
Maíz & $\$ 5.620,00$ & $\$ 14.589,00$ & 259,59 \\
Plátano & $\$ 360,00$ & $\$ 4.474,50$ & $1.242,92$ \\
Naranja & $\$ 3.280,00$ & $\$ 8.037,00$ & 245,03 \\
Otros & $\$ 2.090,00$ & $\$ 6.282,50$ & 300,60 \\
Total & $\$ 17.01800$ & $\$ 49.733,00$ & 292,24 \\
\hline
\end{tabular}

Elaborado por: Autores del artículo

Interpretación de tabla 6. Se efectuó un análisis similar para la producción pecuaria, particularmente porque el pequeño productor cafetalero cuenta con una finca diversa, que integra rubros pecuarios, entre los que destacan las aves y los cerdos, aunque algunos (5 productores) cuentan con unas pocas cabezas de ganado.

En la producción pecuaria se identifica un incremento que corresponde al 174\% con relación al ingreso del año 2016, siendo las aves el rubro animal más frecuente entre los productores y por tanto el de mayor ingreso. El incremento de la producción animal en los dos años de estudio, mediante la vinculación-universidad, ha permitido favorecer el incremento de la producción pecuaria.

Tabla 6. Producción animal

\begin{tabular}{lccc}
\hline \multicolumn{1}{c}{ Rubros } & Año 2016 & Año 2018 & Incremento \% \\
\hline Bovino & $\$ 1.800,00$ & $\$ 9.130,50$ & 507,25 \\
Porcinos & $\$ 1.590,00$ & $\$ 3.573,00$ & 224,72 \\
Aves & $\$ 12.268,00$ & $\$ 14.579,00$ & 118,84 \\
\multicolumn{1}{c}{ Total } & $\$ 15.658,00$ & $\$ 27.282,5$ & 174,24 \\
\hline
\end{tabular}

Elaborado por: Autores del articulo

Para determinar la incidencia de la vinculación con la sociedad en la productividad de los escenarios cafetaleros durante el periodo 2016 - 2018, se planteó un análisis de la hipótesis, mismas que se cita a continuación:

Ho: La vinculación con la sociedad no incide en la productividad de café de los escenarios cafetaleros, periodo 2016-2018. Jipijapa - Manabí.

8 UNESUM-Ciencias. Publicación cuatrimestral. Vol. 5, Año 2021, No. 5 (Septiembre-Diciembre) 
Publicación cuatrimestral. Vol. 5, No. 5 (Septiembre-Diciembre), Año 2021. Pág. 1-14 LA VINCULACIÓN CON LA SOCIEDAD DE LA UNESUM

Interpretación de tabla 7. Para el análisis de datos, se elaboró una base de datos, que fue procesada en el software estadístico SPSS Statistics 25. Los datos obtenidos establecen que hay normalidad, el Coeficiente de Asimetría $>0$, e igual situación ocurre con la Curtosis $>0$, lo que no dio pautas a realizar transformación de datos.

Tabla 7. Análisis de normalidad

\begin{tabular}{cccccccc}
\hline Variable & $\mathrm{n}$ & Media & D.E. & Mín & Máx & Asimetría & Kurtosis \\
\hline $\begin{array}{c}\text { Ingre 2016 } \\
\text { Café }\end{array}$ & 60 & 94,47 & 200,45 & 0 & 1200 & 3,57 & 14,46 \\
$\begin{array}{c}\text { Ingre 2018 } \\
\text { Café }\end{array}$ & 60 & 272,5 & 555,37 & 0 & 3900 & 5,23 & 29,13 \\
\hline
\end{tabular}

Elaborado por: Autores del artículo

Para el análisis de homogeneidad de varianza se consideró a Shapiro - Wilk, cuyo nivel de significancia $<0,01$, establece que no hay varianza homogénea.

Interpretación de tabla 8. Los resultados del análisis (Figura 12 y 13), determinan con un valor de significancia $<0,1$, desecha la hipótesis nula y se acepta la de investigación, los ingresos en los años de estudio, son diferentes entre sí, ante lo cual concluimos que la vinculación ha tenido incidencia en el incremento productivo del rubro café en los escenarios cafetaleros que vienen siendo parte de un trabajo de vinculación.

Tabla 8. Resultado Prueba estadística U de Mann Whitney

\begin{tabular}{ccccc}
\hline & \multicolumn{3}{c}{ Rangos } \\
Años & N & Rango promedio & Suma de rangos \\
\hline \multirow{3}{*}{ Ingresos } & 2016 & 60 & 47,47 & 2848,00 \\
& 2018 & 60 & 73,53 & 4412,00 \\
& Total & 120 & & \\
\hline
\end{tabular}

Elaborado por: Autores del artículo

Tabla 9. Resultado significancia Prueba estadística U de Mann Whitney

\begin{tabular}{lc}
\hline \multicolumn{2}{c}{ Estadístico de prueba a. } \\
\hline & ingresos \\
U de Mann-Whitney & $1.018,000$ \\
W de Wilcoxon & $2.848,000$ \\
Z & $-4,322$ \\
Sig. asintótica(bilateral) & $\mathbf{0 , 0 0 0}$ \\
a. Variable de agrupación: Años & \\
\hline
\end{tabular}

Elaborado por: Autores del artículo

\section{DISCUSIÓN}

Las fincas cafetaleras en su mayoría están gerenciadas por jefes de hogar masculino, cuyas edades superan los 60 años, lo que ratifica que las fincas estén administradas por personas adultas mayores; el nivel de escolaridad en un 46 \% solo aprobó la primaria; en cuanto a servicios 
básicos como el agua el $42 \%$ cuenta con agua entubada captada desde vertientes naturales, solo el $10 \%$ dispone de agua potable procesada en plantas potabilizadoras comunitarias.

Santistevan et al., (2014), en su estudio sobre caracterización de fincas cafetaleras, realizado precisamente en la localidad de Jipijapa, coincide con los resultados obtenidos, indicando que existe déficit de servicios básicos y de infraestructura, agregando que la no atención de estos aspectos conllevara a limitar la competitividad y desarrollo del sector cafetalero. En cuando a la producción de café señala, que es la actividad más importante, pero no es la única actividad; sino que se complementa con producción agropecuaria para el autoconsumo, coincidiendo en este aspecto con lo investigado sobre la producción de otros rubros agrícolas y pecuarios.

Burneo. et al., (2017), enuncia que cuando se trabaja directamente con los caficultores a través de las asociaciones se genera impacto social, resultando la manera más adecuada de abordar a la comunidad, ya que implica un contacto directo con la realidad del sector. Indica además que los estudios de caracterización son valiosos pues permiten, a corto y medio plazo, que los caficultores alcancen un mejor nivel de desarrollo y competitividad, agrega que la correlación entre la comunidad y la academia en la que se integran a los estudiantes, docentes y productores en torno a la misma problemática, permite, por el lado de los productores, se convirtieran en sujetos activos y protagonistas de la experiencia, con participación directa de las acciones a la par del equipo de trabajo de la Universidad, concluyendo que la interacción directa y continua de la academia con el sector productivo contribuye al crecimiento de la economía local y nacional.

La FAO, (2020) por su parte cita, que la vinculación es entre otras instancias, la responsable del impulso de la caficultura en el país, mediante proceso de desarrollo de capacidades técnicas y no técnicas, en este sentido, el sector caficultor mexicano coincide en que la vinculación y alianza entre el sector privado y organizaciones gubernamentales, son los que hasta ahora han podido establecer estrategias sólidas que van de la mano con las acciones globales en pro de un futuro sostenible. (https://www.cecodes.org.co. 2017)

Cedeño et al., (2012) indica que la Universidad constituye un potencial agente dinamizador del cambio social, puesto que forma a los futuros profesionales, que en el desarrollo de su trabajo profesional tendrán un efecto directo e indirecto en su entorno natural, social y cultural; agregando que es la extensión universitaria de suma importancia para el desarrollo. Esta última, como institución social, tiene la misión fundamental de conservar, desarrollar y promover la cultura, incluyendo no solo los aspectos científicos, productivos y tecnológicos, sino los valores, sentimientos, tradiciones y raíces históricas de esa sociedad, a través de sus diferentes formas de manifestación para dar respuesta a las necesidades del desarrollo cultural integral en su más amplia acepción.

Calivá, (2013) confirma lo expuesto por Cedeño, indicando que, la extensión es un proceso esencialmente educativo mediante el cual el extensionistas y productores logran en conjunto objetivos relacionados con el aumento de la productividad, la adopción de innovaciones tecnológicas, la capacitación, el fortalecimiento de las organizaciones y el desarrollo personal y de sus comunidades, lo cual es coincidente con los resultados alcanzados en la presente investigación, donde se aprecia un desarrollo no solo del rubro café, sino de la finca de manera integral.

En tal sentido, la universidad, que desde su misión tiene como propósito la generación, difusión y transferencia de conocimiento a la sociedad, tiene un rol protagónico. Esto implica la necesidad de enfatizar la importancia de la función de extensión universitaria, por ser esta la que se encarga directamente de la interacción con el entorno (Tinoco y Vizarreta, 2014). Además de convertir las

10 UNESUM-Ciencias. Publicación cuatrimestral. Vol. 5, Año 2021, No. 5 (Septiembre-Diciembre) 
estrategias y esfuerzos en resultados productivos tangibles de los micro productores agropecuarios (Moreira et al., 2019).

Moreira et al., (2019) además indica que es importante establecer las relaciones teóricas y prácticas entre la cultura de producción y la vinculación universitaria, pues esta obedece a la necesidad de hacer evidente el encargo de las IES en el logro de conocimientos, desarrollo de habilidades y educación en valores hacia lo laboral, hacia el trabajo.

Al inicio del proyecto se estimuló a los escenarios cafetaleros a ser parte de un proceso organizativo- productivo fundamentado en la vinculación UNESUM - PRODUCTORES, con acompañamiento técnico y social, incentivando progresivamente el interés del desarrollo local, actualmente los pequeños productores por sí mismos solicitan la vinculación.

\section{CONCLUSIONES}

Los escenarios cafetaleros, son la integración de varias familias caficultoras ubicadas en el área rural del cantón Jipijapa que poseen pequeñas fincas (UPAs) diversificadas, teniendo como referencia la producción de café como la actividad económica más importante, se complementan con otras producciones agrícolas para el autoconsumo como plátano, maíz, naranja, y en la producción pecuaria las aves y cerdos.

Los escenarios cafetaleros refieren una media de edad de 56,8 años, con fincas dirigidas por jefes de hogar masculino, con un nivel de escolaridad de un $55 \%$ con solo primaria, con promedio 6,24 integrantes por hogar. En cuanto a servicios básicos, disponen de agua potable solo el $10 \%$, y un $41 \%$ que cuenta con agua de pozo o vertientes; el estado de las viviendas en un $47 \%$ es regular y un 23 \% es mala; el 82\% no dispone de servicio de recolección de basura, y el 100 \% no cuenta con alcantarillado, si disponen de energía eléctrica y servicio telefónico celular.

En cuanto a la descripción del comportamiento productivo de los escenarios cafetaleros el análisis obedeció no solo a aspectos productivos relacionados al plátano, maíz, naranja, y por su puesto el cultivo de café, que alcanzo un incremento del de 288,4 \% en los 2 años de vinculación universitaria. En cuanto a la producción pecuaria, destacan las aves y los cerdos, el incremento total, aunque menor que la producción agrícola, también resulta significativo con un 174,24 \%. Esto indica un cambio a nivel de ingresos, que resulta motivante para el productor.

Se determinó la incidencia de la vinculación universitaria en la productividad de los escenarios cafetaleros durante el periodo 2016 - 2018, mediante la aplicación estadística del test de MannWhitney-Wilcoxon, prueba no paramétrica, aplicada cuando lo datos continuos no expresan normalidad. Los resultados determinaron que la vinculación universitaria por parte de la UNESUM, si ha incidido en el incremento productivo del rubro café. Se cita, que este proceso vinculatorio tuvo como protagonistas a los escenarios cafetaleros, docentes y estudiantes de la Carrera de Agropecuaria y de la Facultad de ciencias Naturales y de la Agricultura.

\section{REFERENCIAS BIBLIOGRÁFICAS}

Andrade Ana. (2017). Análisis y perspectiva de las empresas ecuatorianas exportadoras de productos industrializados de café, periodo 2009 - 2015.

Barrera Fernando, Escarramán Amadeo, Rodríguez César. (2019). Guía para el extensionista del sector cafetalero dominicano. Instituto Interamericano de Cooperación para la Agricultura. ISBN: 978-92-9248-872-7. Obtenido de: http://www.iica.int

Borroto Leal.(2018).La gestión de la vinculación con la sociedad en el Ecuador: REUVIC CASO UNESUM. $11^{\circ}$ Congreso Internacional de Educación Superior Universidad. 2018. ISBN 978-959-16-3243-2. Obtenido de: https://www.caces.gob.ec/wp-content/uploads/2020/02/PONENCIA-DR.-OMELIOBORROTO.

(C) Universidad Estatal del Sur de Manabí. Jipijapa, Ecuador. 
Tomas R Fuentes Figueroa, Kevin J Delgado Pinargote, William A Merchán García

Burneo Valdivieso, J. I.; Capa Mora, E. D.; Jiménez Álvarez, L. S. y Ochoa Cueva, P. A. (2017). Caficultura, un espacio de encuentro entre la academia y el sector productivo. Revista +E versión en línea,7(7), 290-295. Santa Fe, Argentina: Ediciones UNL.

Cadena Iñiguez Pedro, Guevara Francisco, Argüello Raúl, Rendón Roberto. (2018). Proceso de comunicación, extensionismo y adopción de tecnologías. Revista Mexicana de Ciencias Agrícolas volumen 9 número 416 de mayo - 29 de junio, 2018

Calivá E., Juan. (2013). Buenas prácticas de extensión para capacitar, organizar y transferir tecnologías a los productores de café / San José, C.R.: IICA, 2013.84 p.; 17.78 x 25.4 cm.

Cano Menoni Agustín. (2017). La extensión universitaria y la universidad latinoamericana: hacia un nuevo "orden de anticipación” a 100 años de la revuelta estudiantil de Córdoba. Revista +E versión en línea,7(7), 6-23. Santa Fe, Argentina: Ediciones UNL.Dialnet.

Campana Castillo J. (2018). Cultivo de café y modos de vida local: Caso familias del cantón Puyango de la provincia de Loja. Pontificia Universidad Católica del Ecuador.

Cedeño Ferrín, Julio, \& Machado Ramírez, Evelio Felipe. (2012). Papel de la Extensión Universitaria en la transformación local y el desarrollo social. Humanidades Médicas, 12(3), 371-390. Recuperado en 16 de octubre de 2020, de http://scielo.sld.cu/scielo.php?script=sci_arttext\&pid=S1727$81202012000300002 \& \operatorname{lng}=\mathrm{es} \& \operatorname{tlng}=\mathrm{es}$.

CONSEJO CAFETALERO NACIONAL (COFENAC). (2014). Áreas potenciales para el cultivo de coffeacanephora en Ecuador. Informe técnico. Manta: COFENAC.

Consejo de Aseguramiento de la Calidad de la Educación Superior CACES (2020). Educación Superior y sociedad. ¿Qué pasa con la vinculación con la Sociedad? Quito

CONEA (2009). Evaluación del desempeño institucional de las universidades y escuelas politécnicas del Ecuador. Quito: CONEA.

MINISTERIO DE AGRICULTURA Y GANADERÍA. COSTA RICA. (MAG). (2008). Agrocadena - Café. San José.

De Aparicio, Xiomara, ChinininMacanchi, Marco A., \& Toledo Rodríguez, Odalys del Carmen. (2017). El rol de la Vinculación en la Integración de las Funciones Sustantivas de la Universidad Metropolitana del Ecuador. Revista Universidad y Sociedad, 9(4), 37-43. Recuperado en 09 de octubre de 2020, de http://scielo.sld.cu/scielo.php?script=sci_arttext\&pid=S2218-36202017000400005\&lng=es\&tlng=es.

De la Cruz, C., \& Santos, P. (2008). La responsabilidad de la universidad en el proyecto de construcción de una sociedad. Revista Educación Superior y Sociedad, 13(2), 17-52. Recuperado de http://www.izt.uam.mx/spring/wp-content/uploads/2013/11/Respons-de-la-Univ-en-el-proy-de-constr-de-unasoc.pdf

Díaz, C. Herrera, L. (2004). Extensión universitaria. En: Políticas de Estado para la Universidad argentina: balance de una gestión en el nuevo contexto nacional e internacional (pp. 111-117). Recuperado el 11 de febrero de 2011 de: http://www.educ.ar/educar/lm/1192812847436/kbee:/educar/content/portal-content/taxonomiarecursos/recurso/ec45ee6d-0d82-4a59-9591-b0e4d6749b7d.recurso/9b4dff31-a3f5-4fff-99a8cda0af6e2ca1/extension_uni-versitaria.pdf

Duicela, L. (2016). Investigación y desarrollo cafetalero en el Ecuador: Situación actual y perspectivas. Ecuador: Centro de Investigación de Ecuador (CIDE).

FAO. (2020). Pequeñas y pequeños caficultores se vinculan a más mercados nacionales y se preparan para la comercialización con mercados internacionales. Obtenido de: http://www.fao.org/in-action/tropicalagriculture-platform/news/detail/en/c/1271099/

Gaitán Carlos y Pachón Fabio. (2010). Causas para la adopción de tecnologías para la renovación de cafetales - Caso El Colegio (Cundinamarca). Economía y desarrollo rural. Agronomía Colombiana, Vol. 28, Núm. 2

Higuera Inocencio, Rivera Javier. (2018). Chiapas: Problemáticas del sector cafetalero. Centro de Investigación y Asistencia en Tecnología y Diseño del Estado de Jalisco A.C. (CIATEJ)

Instituto Nacional de Estadísticas y Censos. (INEC). (2016). Ecuador en Cifras. Dirección de Estudios Analíticos Estadísticos (DESAE). Quito: INEC, Dirección de Estudios Analíticos Estadísticos (DESAE).

Izaguirre Remón Rafael C., Ortiz Maikel J. Alba Martínez Dixan (2019). Ensayo La extensión universitaria como proceso pedagógico enfocado al desarrollo local. ROCA. Revista científico-educacional de la provincia Granma. Vol.15 No. 2, abril-junio2019. ISSN: 2074-0735. RNPS: 2090.

Martínez de Carrasquero, Mavárez Cynthia, Ramón J, Rojas, P, Ligibther A, \& Carvallo, Belkis. (2008). La responsabilidad social universitaria como estrategia de vinculación con su entorno social. Frónesis, 15(3), 81-

12 UNESUM-Ciencias. Publicación cuatrimestral. Vol. 5, Año 2021, No. 5 (Septiembre-Diciembre) 
103. Recuperado en 10 de octubre de 2020, http://ve.scielo.org/scielo.php?script=sci_arttext\&pid=S131562682008000300006\&lng=es\&tlng=es.

Mato, Daniel. (2013). Contribución de experiencias de vinculación social de las universidades al mejoramiento de la calidad académica y factores que limitan su desarrollo y valoración institucional. Avaliação: Revista da Avaliação da Educação Superior (Campinas), 18(1), 151-180. https://doi.org/10.1590/S1414$\underline{40772013000100009}$

Modelo de Vinculación Universidad Estatal del Sur de Manabí. (2017)

Moreira Vera D., Zambrano Cedeño J., Machado Ramírez Evelio F. (2019). Fundamentos del proceso de vinculación universitaria en la formación de una cultura de producción. DOI: https://doi.org/10.35195/ob.v11i3.795. Revista Opuntia Brava Volumen: 11 Número: 3. ISSN. 2222-081X

Ortiz Riaga M. Morales Rubiano M. (2011). La extensión universitaria en América Latina: concepciones y tendencias. Universidad de La Sabana. Facultad de Educación. ISSN 0123-1294 | Educ.Educ. Vol. 14, No. 2. pp. 349-366.

Pérez Matute V. Villacís Andrade D. (2015). Análisis del proyecto de reactivación de la caficultura ecuatoriana en la zona de Manabí. Obtenido de: http://repositorio.ucsg.edu.ec/bitstream/3317/4164/1/T-UCSG-PRE-ESP-CFI179.pdf

PDOT Jipijapa. (2015). Actualización del Plan de Ordenamiento Territorial Jipijapa. Obtenido de:http://app.sni.gob.ec/sni-

link/sni/PORTAL_SNI/data_sigad_plus/sigadplusdocumentofinal/1360000630001_PDYOT\%20JIPIJAPA\%2 014042016_14-04-2016_10-45-27.pdf

Polaino J., Cecilia; Romillo, Antonio de J. (2017). Vinculación con la Sociedad en la Universidad de Otavalo, Ecuador Formación Universitaria, vol. 10, núm. 3, pp. 21-30 Centro de Información Tecnológica. La Serena, Chile

Ponce Vaca Luciano, Orellana Kleber, Acuña Isidro. (2016). Diagnóstico y propuesta de un sistema de innovación tecnológica cafetalera en Ecuador. Revista Cubana de Ciencias Forestales. Volumen 4, número 2

Ponce Vaca, Luciano Abelardo, Orellana Suarez, Kléber Dionicio, Acuña Velásquez, Isidro Rolando, Alfonso Alemán, Juan Luis, \& Fuentes Figueroa, Tomas. (2018). Situación de la caficultura ecuatoriana: perspectivas. Revista Estudios del Desarrollo Social: Cuba y América Latina, 6(1), 307-325. Recuperado el 05 de octubre de 2020, de http://scielo.sld.cu/scielo.php?script=sci_arttext\&pid=S230801322018000100015\&lng=pt\&tlng=es

Pozo Mónica. (2014). Análisis de los factores que inciden en la producción de café en el Ecuador 2000 -2011. Pontificia Universidad Católica del Ecuadorfacultad de Economía.

Reglamento al régimen académico (2019). Vinculación con la sociedad, prácticas preprofesionales y redes académicas. Quito.

Romero Cantarero R. (2018). Aliados estratégicos de la universidad en los procesos de vinculación con la sociedad. Universidad Nacional Autónoma de Honduras. Congreso Universidad. $11^{\circ}$ Congreso Internacional de Educación Superior Universidad. 2018. ISBN 978-959-16-3243-2.

Rivera Ramón; Rueda Hernández; Ramírez Liberio. (2019). Practica educativa y responsabilidad social. Servicios Académicos Intercontinentales para eumed.net. Universidad de Málaga, Málaga, España. 2019 ISBN-13: 97884-17583-55-2. Obtenido de: https://www.eumed.net/libros/1833/1833.pdf

Rojas Cairampoma, Marcelo. (2015). Tipos de Investigación científica: Una simplificación de la complicada incoherente nomenclatura y clasificación. REDVET. Revista Electrónica de Veterinaria, vol. 16, núm. 1, 2015, pp. 1-14. Veterinaria Organización Málaga, España

Santamaria Velasco Carlos. (2011). La creación de empresas en el sistema universitario mexicano. Tesis Doctoral. Universidad Rovira i Virgili.

Santistevan Méndez M; Julca Otiniano A; Borjas Ventura R; Tuesta Hidalgo O. (2014). Caracterización de fincas cafetaleras en la localidad de jipijapa (Manabí, Ecuador). Ecología Aplicada, 13(2). ISSN 1726-2216. Obtenido de: https://www.recimundo.com/index.php/es/article/download/218/html?inline=1

Simbaña Cabrera H, Correa Elisabeth. (2018). La vinculación con la sociedad en el Ecuador: Reflexiones sobre su itinerario curricular. Revista Vínculos, 2(1). doi:http://dx.doi.org/10.24133/rvespe.v2i1.563

Tinoco Gómez, Oscar; Vizarreta Chía, Roberto. (2014). Extensión universitaria, proyección social y su relación con la investigación y formación profesional en el marco del proceso de acreditación universitaria en la FII 
Industrial Data, vol. 17, núm. 1, enero-junio, 2014, pp. 39-45Universidad Nacional Mayor de San Marcos Lima, Perú

Tommasino, Humberto; Rodríguez, Nicolás. (2010). Tres tesis básicas sobre extensión y prácticas integrales en la Universidad de la República, bases y fundamentos.

Tünnermann, C. (2000). El nuevo concepto de la extensión universitaria y difusión cultural y su relación con las políticas de desarrollo cultural en América Latina. En: Anuario de Estudios Centroamericanos, 4: 93-126

Unesco (1998). Declaración mundial sobre la educación superior en el siglo XXI: visión y acción. En: Conferencia Mun-dial sobre Educación Superior. París, Francia.

Venegas Sánchez S; Orellana Bueno D; Pérez Jara P. (2018). La realidad ecuatoriana en la producción de café. Revista Científica de Investigación actualización del mundo de las Ciencias. Vol. 2 núm., 2, mayo, ISSN: 2588-073X, 2018, pp. 72-91. DOI:10.26820/recimundo/2.(2).2018.24-44. Obtenido de: https://www.recimundo.com/index.php/es/article/download/218/html?inline=1 